\title{
ON THE FUNDAMENTAL GROUP OF A COMPACT NEGATIVELY CURVED MANIFOLD
}

\author{
SU-SHING CHEN
}

\begin{abstract}
A question of Hirsch and Thurston on the fundamental group of a compact negatively curved manifold is investigated.
\end{abstract}

1. In [8], Hirsch and Thurston have asked the question whether the fundamental group $\pi_{1}(M)$ of a compact Riemannian manifold $M$ of negative sectional curvature is in the class $C$ of groups which contains all amenable groups and which satisfies: if $G$ and $H$ are in $C$, then the free product $G^{*} H$ is in $C$, and if $G$ has finite index in $K$ then $K$ is in $C$. This question is related to their investigation [8] on the Euler characteristic $\chi(M)$ of $M$. In this paper, we shall give some evidence for $\pi_{1}(M)$ of a compact Riemannian manifold $M$ of negative sectional curvature not in this class $C$ of groups. According to [8], $\pi_{1}(M)$ in $C$ would imply that the Euler characteristic $\chi(M)$ vanishes. Consequently, our result to a certain extent supports the truth of a wellknown conjecture that for a compact negatively curved manifold $M$ of even dimension $n, \chi(M)<0$ for $n=2 \bmod 4$ and $\chi(M) \geqslant 0$ for $n=0 \bmod 4$.

We first show that any amenable subgroup of $\pi_{1}(M)$ is infinite cyclic. If $M$ has nonpositive sectional curvature, then it is not known whether any amenable subgroup of $\pi_{1}(M)$ is a finite extension of abelian groups. When the subgroup is solvable, this is known to be true [7], [12].

Next, we show the following statements:

(1) $\pi_{1}(M)$ has relations for a certain set of generators. ${ }^{1}$

(2) A subgroup of $\pi_{1}(M)$ with an amenable subgroup of axial elements of finite index is an amenable group of axial elements and is infinite cyclic.

(3) A free product of amenable subgroups of axial elements is free.

(4) A subgroup of $\pi_{1}(M)$ with a free product subgroup of amenable subgroups of axial elements of finite index is free.

(5) If $\pi_{1}(M)$ belongs to the class $C$, then $\pi_{1}(M)$ is free. We shall use a deep result of Stallings [13] and Swan [14] to prove statement (4). Stallings has proved that a finitely generated torsion free group with a free subgroup of finite index is free [13]. In [14], Swan has extended this theorem to infinitely generated groups. A direct proof of statement (4) seems to be possible.

Received by the editors June 24, 1977 and, in revised form, October 14, 1977 and November 3, 1977.

AMS (MOS) subject classifications (1970). Primary 53C20, 53C35.

Key words and phrases. Negative sectional curvature, fundamental group.

${ }^{1}$ This statement is not enough to imply that $\pi_{1}(M)$ is not a free group.

c) American Mathematical Society 1978 
2. Let $M$ be a compact negatively curved manifold. Then the sectional curvature is bounded from above by a negative constant. The group $G$ of covering transformations associated to the universal covering $\tilde{M}$ of $M$ is isomorphic to $\pi_{1}(M) . G$ is the disjoint union of its stability groups $G_{x}$, $x \in \tilde{M}(\infty)$ [5]. Here $\tilde{M}(\infty)$ denotes the boundary of $\tilde{M}$ [5]. All elements of $G$ are axial and they translate certain geodesics [3]. There are exactly two fixed points in $\tilde{M}(\infty)$ of each axial elements. Each $G_{x}$ is infinite cyclic. The limit set $L(G)$ of $G$ is $\tilde{M}(\infty)$.

THEOREM 1. Let $M$ be a compact negatively curved manifold. Any amenable subgroup of $\pi_{1}(M)$ is infinite cyclic.

Proof. Let $H$ be any subgroup of $G$. The limit set $L(H)$ of $H$ is one of the following [5]: (1) one point and every element of $H$ is parabolic, (2) two points and $H$ is infinite cyclic of axial elements, (3) a perfect nowhere dense set in $\tilde{M}(\infty)$ and (4) $\tilde{M}(\infty)$. It is clear that (1) cannot occur. If (3) and (4) occur, then we may use Eberlein's freeness argument [4] to show that there is a free subgroup $F$ of at least two generators of $H$. Hence $H$ cannot be amenable. Thus $H$ is infinite cyclic and consists of axial elements of the same fixed points.

COROLlary. Every abelian or solvable subgroup of $\pi_{1}(M)$ is infinite cyclic (see [1], [11]).

THEOREM 2. Let $M$ be a compact negatively curved manifold. There is at least one relation for a certain set of generators of $\pi_{1}(M)$.

Proof. Let $G$ be a discrete group of isometries of $\tilde{M}$. One can form the Dirichlet fundamental region $\Re$ for $G$ in $\tilde{M}$. $\Re=\left\{p \in \tilde{M} \mid d\left(p, p_{0}\right)<\right.$ $d\left(p, \phi\left(p_{0}\right)\right), \forall \phi \in G$ and $\left.\phi \neq \mathrm{id}\right\}$. Here $p_{0}$ is a point not fixed by $G$. Then the following properties are known [6]:

(1) Every point in $\tilde{M}$ is identified with exactly one point of $\Re$ or finitely many points of the boundary $\mathrm{Bd}(\Re)$.

(2) $\Re$ is not empty and $\mathrm{Bd}(\Re)=\left\{p \in \dot{\dot{M}} \mid d\left(p, p_{0}\right) \leqslant d\left(p, p_{j}\right), \forall j>0\right.$ and $d\left(p, p_{0}\right)=d\left(p, p_{i}\right)$ for some $\left.i>0\right\}$, where $G=\left\{\phi_{i}\right\}$ and $p_{i}=\phi_{i}\left(p_{0}\right)$, $i>0$.

(3) Each compact subset $K$ of $\tilde{M}$ meets only finitely many images $\phi(\bar{\Omega})$, $\phi \in G$ of $\Re$ under $G$. The set $S_{i}=\bar{\Re} \cap\left\{p \in \tilde{M} \mid d\left(p, p_{0}\right)=d\left(p, p_{i}\right)\right\}$ is called the side of $\Re$ determined by $\phi_{i}$. If $S_{i}$ is the side of $\Re$ determined by $\phi_{i}$ and $S_{i}^{\prime}$ is the side of $\Re$ determined by $\phi_{i}^{-1}$, then $S_{i}$ is conjugate to exactly one other side $S_{i}^{\prime}$ of $\Re$.

Since $M$ is compact, the Dirichlet region $\Re$ for $G$ in $\tilde{M}$ is compact and consists of finitely many sides which intersect at lower dimensional faces. Let $T$ be a lower dimensional face in $\operatorname{Bd}(\Re)$ which lies on some side $S_{k_{1}}$ determined by $\phi_{k_{1}} \in G$. Then $S_{k_{1}}$ is conjugate to $S_{k_{1}}^{\prime} \cdot \phi_{k_{1}}(T)$ lies on $S_{k_{1}}^{\prime}$ and some other side $S_{k_{2}}$ determined by $\phi_{k_{2}}$. Then $\phi_{k_{2}} \phi_{k_{1}}(T)$ lies on $S_{k_{2}}^{\prime}$ and some other side. Consider an orbit of $T$ under these conjugations. Then this orbit 
must be finite and is $\left\{T, \phi_{k_{1}}(T), \ldots, \phi_{k_{t}} \phi_{k_{t-1}} \cdots \phi_{k_{1}}(T)=T\right\}$. Since $\phi_{k_{1}} \phi_{k_{t-1}} \cdots \phi_{k_{1}}$ is axial and leaves a proper subset $T$ of $M$ invariant, it has to be the identity. Thus $G$ has a relation.

3. We need a theorem due to Swan [14] which generalizes a theorem of Stallings [13].

THEOREM (SWAN). A torsion free group (finitely or infinitely generated) with a free subgroup $F$ of finite index is a free group.

Proposition 1. A subgroup of $\pi_{1}(M)$ with an amenable subgroup of axial elements of finite index is an amenable subgroup of axial elements and is infinite cyclic.

Proof. Since the limit set $L(H)$ of $H$ with an amenable subgroup $G_{x}$ $(x \in \tilde{M}(\infty))$ of axial elements of finite index is equal to the limit set $L\left(G_{x}\right)$, $L(H)$ consists of two points and is an infinite cyclic group of axial elements.

Proposition 2. A free product of amenable subgroups of axial elements is a free group.

Proof. Each amenable group of axial elements is infinite cyclic. The given free product is free from the definition of free products.

Proposition 3. A subgroup of $\pi_{1}(M)$ with a free product subgroup of amenable subgroups of axial elements of finite index is a free group.

Proof. Since $\pi_{1}(M)$ is torsion free, the given group is also. We may apply Swan's theorem to obtain the statement.

REMARK. Can one give a geometric proof of the above proposition?

THEOREM 3. Let $M$ be a compact negatively curved manifold. If $\pi_{1}(M)$ belongs to the class $C$ of groups then $\pi_{1}(M)$ is free.

Proof. Each group in $C$ is obtained by a composition of operations described in Propositions 1, 2 and 3. The resulting group is a free group.

\section{REFERENCES}

1. W. Byers, On a theorem of Preissmann, Proc. Amer. Math. Soc. 24 (1970), 50-51.

2. S. Chen, Complete homogeneous Riemannian manifolds of negative sectional curvature, Comment. Math. Helv. 50 (1975), 115-122.

3. __ A remark on a question of Margulis, Duke Math. J. 43 (1976), 805-808.

4. P. Eberlein, Some properties of the fundamental group of a fuchsian manifold, Invent. Math. 19 (1973), 5-13.

5. P. Eberlein and B. O'Neill, Visibility manifolds, Pacific J. Math. 46 (1973), 45-109.

6. P. E. Ehrlich, The Dirichlet fundamental region and elliptic isometries for Riemannian manifolds without conjugate points (preprint).

7. D. Gromoll and J. A. Wolf, Some relations between the metric structure and the algebraic structure of the fundamental group in manifolds of nonpositive curvature, Bull. Amer. Math. Soc. 77 (1971), 545-552.

8. M. W. Hirsch and W. P. Thurston, Foliated bundles, invariant measures and flat manifolds, Ann. of Math. 101 (1975), 369-390.

9. J. Lehner, Discontinuous groups and automorphic functions, Math. Surveys, no. 8, Amer. Math. Soc., Providence, R.I., 1964. 
10. T. S. Motzkin, B. O'Neill and E. G. Straus, Isolated subgroups, Michigan Math. J. 20 (1973), 235-248.

11. A. Preissmann, Quelques propriétés globales des espaces de Riemann, Comment. Math. Helv. 15 (1942-43), 175-216.

12. S. T. Yau, On the fundamental group of compact manifolds of nompositive curvature, Ann. of Math. 93 (1971), 579-585.

13. J. Stallings, Group theory and three-dimensional manifolds, Yale Math. Monographs No. 4, Yale University, New Haven, Conn., 1971.

14. R. G. Swan, Groups of cohomological dimension one, J. Algebra 12 (1969), 588-610.

Department of Mathematics, University of Florida, GainesViLle, Florida 32611 